ORIGINAL ARTICLE

\title{
The relation of infantile spasms, tubers, and intelligence in tuberous sclerosis complex
}

\author{
F J K O'Callaghan, T Harris, C Joinson, P Bolton, M Noakes, D Presdee, S Renowden, A Shiell, \\ C N Martyn, J P Osborne
}

See end of article for authors' affiliations

....................

Correspondence to:

Dr F J K O'Callaghan,

Department of Paediatric

Neurology, Bristol Royal

Hospital for Children,

Institute of Child Health,

Upper Maudlin Street,

Bristol BS2 8BJ, UK; finbar.

ocallaghan@ubht.nhs.

swest.uk

Accepted

27 September 2003

\begin{abstract}
Background: The aetiology of the learning difficulty in tuberous sclerosis is debated. It may be related to the amount of tubers in the brain or caused by the infantile spasms that occur in early life.

Aims: To examine the relative contributions to final intelligence (IQ) made by both cerebral tubers and infantile spasms.

Methods: As part of an epidemiological study of tuberous sclerosis in the south of England, patients were recruited who were able to undergo magnetic resonance imaging (MRI) without the need for an anaesthetic. Epilepsy history was determined by interview and review of clinical records. IQ was assessed using either Wechsler intelligence scales or Raven's matrices.

Results: A total of 41 patients consented to have an MRI scan. IQ scores were normally distributed about a mean of 91. Twenty six patients had a positive history of epilepsy, and 11 had suffered from infantile spasms. There was a significant relation between the number of tubers and IQ. Infantile spasm status partly confounded the relation between tubers and IQ, but did not render the relation statistically insignificant. The relation between infantile spasms and learning difficulty remained strong even when controlling for the number of tubers.
\end{abstract}

$\mathrm{T}$ he tuberous sclerosis complex (TSC) is a genetic disease in which benign tumours (hamartomas) may develop in multiple organ systems. Approximately three quarters of TSC patients have epilepsy, and half will have suffered from a form of epileptic seizures in the first year of life known as infantile spasms. ${ }^{1}$ The association of the disease with mental retardation has been recognised since the first case was described by Bourneville in $1880^{2}$ and has been cemented in the minds of clinicians since 1908 when Vogt described a triad of features (adenoma sebaceum, now called facial angiofibromatosis, epilepsy, and mental retardation) that he felt characterised the disease. ${ }^{3}$ Recent epidemiological studies have shown that cognitive impairment is less common in TSC than was first thought and occurs in just under half of cases. ${ }^{4}$ However, learning difficulties are often severe and are still one of the most devastating consequences of the condition.

Infantile spasms are a rare form of epilepsy that occurs predominantly in the first year of life. There are multiple aetiologies including malformations and acquired insults to the developing brain. However, in some infants no cause is found. TSC is the cause of infantile spasms in 10-20\% of those affected. All those affected, regardless of aetiology, have a very high risk of severe learning difficulties and continuing epilepsy. Diagnosis is frequently delayed because of the subtle onset of seizures in many infants and the effect, if any, of this delay is unknown. There is controversy about the best way to manage infantile spasms because there is inadequate information on the relative effectiveness of different treatments and the risks of serious side effects from therapy.

In TSC, two main hypotheses have been proposed to explain the development of the intellectual impairments. The first is that anatomical pathology, indexed by the number of cerebral hamartomas (tubers) in the brain, determines intelligence level (IQ). ${ }^{5-12}$ The second is that the infantile spasms are the critical determinant of future intellectual function in these patients. ${ }^{13-15}$ Supporters of the second hypothesis point to cases of children who had apparently developed normally until the onset of spasms, at an age when normal development was well advanced and when new tubers are thought not to occur, whereupon their development regressed significantly and was left permanently affected.$^{16}$ It is rare for an individual with TSC to have severe learning difficulties in the absence of a history of early onset epilepsy. Previous studies have shown a strong association between learning difficulty and the presence of epilepsy, but in particular a history of infantile spasms. ${ }^{1}{ }^{14}{ }^{17-19}$ If infantile spasms are part of the aetiology of learning difficulties, then treating (or even preventing) them becomes even more important. In addition, it might suggest that the treatment of infantile spasms in other conditions should also be a matter of urgency.

Of course, the two aetiological hypotheses are not mutually exclusive. For example, infantile spasms could lie on the causal pathway between cerebral pathology and subsequent intellectual impairment. Surprisingly, no one has previously looked at the relative contribution of both variables to subsequent intelligence in the same group of patients. In this study we assess the intelligence of a sample of TSC patients and look at the relation between cognitive function and both cerebral pathology (as indexed by tuber count) and infantile spasm status. We assessed cerebral pathology using MRI scans because the number and site of cerebral tubers identified by this imaging modality correlates well with the numbers identified at postmortem examination. ${ }^{20}$ Ethical approval for the study was obtained from the South West Multicentre Research Ethics Committee and the relevant local research ethics committees.

\section{METHODS}

In August 1998 we undertook an epidemiological study of TSC in the South of England. A total of 179 cases were identified in a population of 3679 162, thus giving a prevalence rate of 4.9 per 100000 (95\% confidence interval 4.2 to 5.6); 149 patients and their carers agreed to be interviewed and examined. Specific enquiries were made 
about early developmental history and history of seizures. Clinical case notes were reviewed. Individuals were classified as either having, or not having, a positive history of infantile spasms. Intelligence was assessed using the Wechsler Adult Intelligence Scale (WAIS-R, Psychological Corporation), the Wechsler Intelligence Scale for Children (WISC-III, Psychological Corporation), or Raven's coloured matrices (Raven's Coloured Progressive Matrices, Psychological Corporation), depending on the age and ability level of the individual. Patients who were able to undergo magnetic resonance imaging (MRI) without the need for sedation or an anaesthetic (that is, $>7$ years of age and with an IQ > $>50$ ) were invited to have a cranial MRI. All scans were performed at the Royal United Hospital, Bath using a Siemens Magnetom Impact Expert 1.0 tesla MRI scanner. All patients underwent the same imaging protocol that included T1, T2, and fluid attenuated inversion recovery (FLAIR) sequences. The scans were reported by a neuroradiologist using a standardised reporting format. The radiologist was asked to record the numbers and site of tubers. She was blinded to the name and clinical status of the patient. Comparisons between groups were made using Mann-Whitney U tests. The relation between numbers of cerebral tubers, infantile spasm status, and cognitive outcome was explored using multiple linear regression. The univariate relations between tuber counts in the individual lobes of the brain and IQ were initially investigated using simple linear regression. Both forward and backward stepwise linear regression was used to investigate the relative contributions made by tuber count in each lobe to final IQ score. The statistical software package STATA (version 7.0) was used for all analyses.

\section{RESULTS}

Forty one patients consented to have an MRI scan. There were 24 females and 17 males. Ages ranged from 9 to 75; the distribution of ages was skewed to the right, with a median of 25 years (interquartile range 17-46). All 41 patients completed the Raven's coloured matrices and 33 patients completed the Wechsler assessments. We used the Wechsler scores where possible in the analysis, but in the eight patients who did not have a WISC or WAIS score we used the IQ derived from the Raven's matrices. The IQ scores for this subset of patients were normally distributed about a mean of 91 (range 52-130, standard deviation $=18.8$ ). Twenty six patients $(63 \%)$ had a positive history of epilepsy, and 11 (25\%) had suffered from infantile spasms.

All patients had evidence of at least one intracranial tuber. The distribution of tubers was skewed to the right, ranging from 1 to 32 (median $=10$, interquartile range $5-16)$. Tubers were most commonly seen in the frontal lobes ( 37 patients), followed in order by the parietal (33 patients), occipital (30 patients), temporal lobes (16 patients), and cerebellum (4 patients).

Figure 1 shows the scatter plot showing the relation between the number of tubers and intellectual performance in the 41 patients. The relations were explored further using multiple regression techniques. The basic regression model showed that there was a significant relation between the number of tubers and IQ (regression coefficient $=-1.2$; standard error $=0.27, \mathrm{p}<0.001 ; \mathrm{y}=105.5-1.2 \mathrm{x} ; \mathrm{R}^{2}=0.33$ ). Neither age nor sex was significantly related to IQ or tuber count. However, a history of infantile spasms was significantly related with IQ. Those patients with a positive history of infantile spasms had a mean IQ score of 70.7 compared to 97.3 in the other patients $(p=0.0005$, Mann-Whitney test). The median tuber count was also higher in those patients with infantile spasms (20) than those without a history of spasms $(8, p=0.0016$, Mann-Whitney test). The inclusion of infantile spasm status into the regression model does partly confound the relation between tubers and IQ, reducing the regression coefficient from -1.2 to -0.75 , but does not render the relation statistically insignificant (regression coefficient $=-0.75 ; \quad$ standard $\quad$ error $=0.3, \quad p=0.016$; $y=104.4-0.75 x_{1}-18.1 x_{2} ; R^{2}=0.47$, where $x_{1}=$ tuber count and $\mathrm{x}_{2}=$ infantile spasm status). Similarly the relation between infantile spasms and learning difficulty remained strong even when controlling for the number of tubers in the patients' brains (regression coefficient $=18.1$; standard error $=6.2, p=0.006)$. Tubers alone accounted for $33 \%$ of the variance in IQ scores and adding infantile spasm status generated a regression model that accounted for $47 \%$ of the variance in IQ.

There were strong correlations between numbers of tubers in each of the individual lobes of the brain and between numbers of tubers in each individual lobe and IQ score (see table 1). Tuber counts in the frontal, temporal, and occipital lobes were each significantly associated with IQ score in univariate analyses. Both forward and backwards stepwise multiple linear regressions (using p values of 0.1 for inclusion or exclusion from the model) resulted in only frontal and occipital lobe tuber count remaining in the model. Occipital lobe tuber count was the only significant predictor of IQ score $(\mathrm{p}<0.05)$ when tuber counts in the individual lobes were examined together (see table 2 ).

\section{DISCUSSION}

This study shows that in a group of 41 mildly affected TSC patients a history of infantile spasms is strongly associated with a lower intelligence level. This relation is not confounded by age, gender, or tuber number in the brain. Similarly the significant association between tuber count and IQ remains statistically significant even after adjusting for infantile spasm status.

In this cranial imaging study of TSC patients we have imaged all the patients on the same MRI scanner, using the same imaging protocol, and all the films have been reported by a neuroradiologist who was blinded to the clinical status of the patient. However, some caveats need to be mentioned. Firstly, only 41 patients agreed to undergo the research scanning procedure. It is possible therefore that biases may have been introduced by the way that the participants selected themselves for the study. However, the characteristics of the group that were studied, in terms of age, sex, and epilepsy status were very similar to the total population of

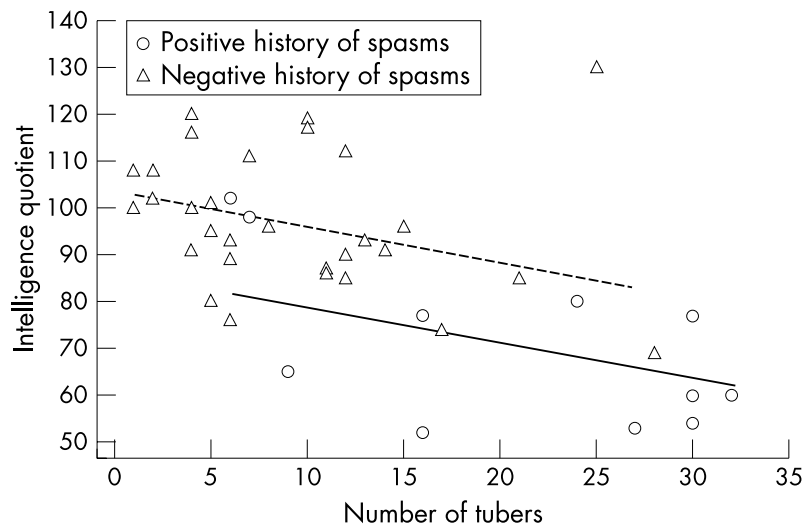

Figure 1 Scatter plot of intelligence quotient scores versus total number of tubers identified on MRI scan. Patients with a negative history of infantile spasms are depicted by a triangle and those with a positive history by a circle. Two regression lines are drawn depicting the linear relation between intelligence quotient and tuber count both with (solid line) and without (dashed line) a positive history of infantile spasms. 
Table 1 Correlation matrix indicating extent of correlation (denoted by correlation coefficient, $r$ ) between numbers of tubers in each individual lobe of the brain

\begin{tabular}{|c|c|c|c|c|c|}
\hline & IQ & $\begin{array}{l}\text { Frontal } \\
\text { lobe }\end{array}$ & $\begin{array}{l}\text { Parietal } \\
\text { lobe }\end{array}$ & $\begin{array}{l}\text { Temporal } \\
\text { lobe }\end{array}$ & $\begin{array}{l}\text { Occipital } \\
\text { lobe }\end{array}$ \\
\hline IQ & 1 & & & & \\
\hline Frontal lobe & $-0.37^{*}$ & 1 & & & \\
\hline Parietal lobe & -0.29 & $0.56^{* *}$ & 1 & & \\
\hline Temporal lobe & $-0.33^{*}$ & $0.55^{\star *}$ & 0.22 & 1 & \\
\hline Occipital lobe & $-0.43^{*}$ & $0.35^{\star}$ & $0.57^{* *}$ & 0.22 & 1 \\
\hline
\end{tabular}

*Correlation coefficient significant at $\mathrm{p}<0.05$ level.

**Correlation coefficient significant at $p<0.001$ level.

normal intellect and mildly disabled TSC patients in the Wessex study.

Secondly, we performed full Wechsler assessments of intelligence in only 33 patients. In the other eight patients we had to rely on an IQ score derived from the Raven's coloured matrices. The matrices are often used to assess the intelligence of children, the elderly, or the intellectually impaired. However, the test may be subject to a ceiling effect when used in adult individuals of above average intelligence. This is unlikely to have been the case in this study. The IQ scores of the eight patients who were only assessed with the Raven's matrices ranged from 60 to $100($ median $=85)$. The correlation between scores on the Raven's matrices and the Wechsler scales in the 33 patients who completed both tests was high (correlation coefficient $=0.83, \mathrm{p}<0.001$ ), and when the analysis was confined to the 33 patients who had the more detailed IQ tests, then both tuber count and infantile spasms status remained statistically significant predictors of intelligence.

Thirdly, we have had to rely on historical data to document epilepsy history. Case notes were inspected for all cases in the study but in no case did we have the benefit of diagnostic tests that might now be considered the gold standard for diagnosing infantile spasms (that is, video-electroencephalography). The diagnosis of infantile spasms was therefore dependent on the diagnostic accuracy of the clinician at the time and it is possible that there may have been some misclassification of epilepsy type. However, it is likely that any misclassification of infantile spasm status would have been non-differential (that is, random), and therefore its effect would be to dilute any positive associations that we now see between spasm status and IQ. The use of historical data also means that it is not possible to assess either the severity or duration of infantile spasms but only whether or not they were present.

This study is consistent with previous findings in this area. Several authors have shown that either the number of tubers or a history of infantile spasms are strongly associated with the presence of learning difficulty in TSC. However, this study is the first that shows that both the number of tubers and a history of infantile spasms are associated with cognitive deficits in TSC patients independently of each other.

We have shown these associations in mildly affected patients. Many previous authors have accepted that the tuber number is greater in patients with severe learning difficulties but may not have been aware that tuber count is associated with gradations of intellect in the normal intellect population of TSC sufferers. However, we would be cautious about making statements about intellectual prognosis for the individual on the basis of tuber number seen on MRI scan. There was one individual in this study who had an IQ of 130 and 27 tubers. Moreover, different scanners and imaging protocols may be more or less sensitive at detecting tubers.
Table 2 Results of forward stepwise multiple linear regression model investigating relation between tuber count in individual lobes of the brain and IQ

\begin{tabular}{lllllll}
\hline $\begin{array}{l}\text { IQ } \\
\text { (=dependent } \\
\text { variable) }\end{array}$ & $\begin{array}{l}\text { Regression } \\
\text { coefficient }\end{array}$ & $\begin{array}{l}\text { Standard } \\
\text { error }\end{array}$ & t value & p value $95 \% \mathrm{Cl}$ \\
\hline $\begin{array}{l}\text { Occipital lobe } \\
\text { tuber count }\end{array}$ & -3.1 & 1.4 & -2.2 & 0.031 & -5.8 to -0.3 \\
$\begin{array}{l}\text { Frontal lobe } \\
\text { tuber count }\end{array}$ & -0.94 & 0.56 & -1.7 & 0.099 & -2.1 to 0.19 \\
Constant & 102.5 & 4.5 & 22.8 & $<0.00193 .4$ to 111.6 \\
\hline
\end{tabular}

Number of observations $=41 ; R^{2}=0.24$; adjusted $R^{2}=0.20 ; F=6.02$; probability $>\mathrm{F}=0.0054$

Beginning with empty model: $p=0.0052$ adding occipital lobe tuber count; $p=0.0989$ adding frontal lobe tuber count.

Goodman et al suggested that having more than seven tubers carried a high risk of intellectual impairment. ${ }^{12}$ However, in our sample the median number of tubers in those patients without severe intellectual impairment was 10.

Although several authors have previously commented on the strong association between infantile spasms and severe learning difficulty, others have pointed out that infantile spasms need not have a dismal prognosis in TSC. ${ }^{21}$ We would agree with both observations, but this study in mildly affected TSC patients shows that infantile spasms, while clearly not associated with severe disability in these patients, were still associated with cognitive deficit.

We have shown that, when analysed separately, tuber count in the frontal, temporal, and occipital lobes are each significantly related to IQ. There are also strong and significant correlations between tuber counts in each of the individual lobes. Consequently, in any regression model that includes tuber counts in each of the individual lobes as separate variables there may be problems of co-linearity which may render the model inherently unstable. The model may also be unstable because it includes multiple variables (that is, five) when the total number of participants in the study is small $(n=41)$. However, we did perform a stepwise linear regression that included separate variables for tuber counts in each individual lobe and this resulted in occipital lobe tuber count alone being a significant predictor of IQ. We have no biologically plausible explanation as to why occipital lobe tuber count may be more strongly associated with IQ score in these patients.

We conclude that both the number of cortical tubers and a history of infantile spasms are strongly associated with IQ in TSC patients. These findings are compatible with both hypotheses for the aetiology of learning difficulty in TSC. It remains possible that infantile spasms are an epiphenomenon of unmeasured cerebral pathology which we have therefore not been able to control for in this study. However, while the possibility remains that infantile spasms may be important in determining the intellectual outcome of individuals with tuberous sclerosis, further research on the effects of therapy on spasms and subsequent intellectual development is required. The challenge remains to find better methods of identification and treatment for this form of epilepsy. Similarly, improved methods of imaging cerebral pathology in TS are required in order to determine more precisely the nature of the relation between structural pathology and the likelihood of learning disabilities developing.

\section{ACKNOWLEDGEMENTS}

The authors would like to thank the Wellcome Trust, Bath Unit for Research in Paediatrics, The Tuberous Sclerosis Association, and the Medical Research Council for their help. F O'Callaghan was 
supported by a Wellcome Trust Fellowship in Clinical Epidemiology for the duration of this study.

\section{Authors' affiliations}

F J K O'Callaghan, Department of Paediatric Neurology, Bristol Royal Hospital for Children, Institute of Child Health, Upper Maudlin Street, Bristol BS2 8BJ, UK

T Harris, C Joinson, P Bolton, Developmental Psychiatry Section, University of Cambridge, Douglas House, $18 \mathrm{~b}$ Trumpington Road, Cambridge CB2 2AH, UK

M Noakes, D Presdee, J P Osborne, Royal United Hospital, Combe Park, Bath BA1 3NG, UK

S Renowden, Department of Neuroradiology, Frenchay Hospital, Bristol, UK

A Shiell, C N Martyn, MRC Environmental Epidemiology Unit, Southampton General Hospital, Tremona Road, Southampton SO16 6YD, UK

\section{REFERENCES}

1 Webb DW, Fryer AE, Osborne JP. Morbidity associated with tuberous sclerosis: a population study. Dev Med Child Neurol 1996;38:146-55.

2 Bourneville DM. Sclerose tubereuse des circonvolutions cerebrales: idiotie et epilepsie hemiplegique. Paris. Arch Neurol 1880;1:81-91.

3 Vogt H. Zur diagnostik der tuberosen sclerose. Z Erforsch Behandl Jugendl schwachsinns 1908;2:1-16.

4 Webb DW, Fryer AE, Osborne JP. On the incidence of fits and mental retardation in tuberous sclerosis. J Med Genet 1991;28:395-7.

5 Kingsley DP, Kendall BE, Fitz CR. Tuberous sclerosis: a clinicoradiological evaluation of 110 cases with particular reference to atypical presentation. Neuroradiology 1986;28:38-46.

6 Inove Y, Nakajima S, Fukuda T, et al. Magnetic resonance images of tuberous sclerosis. Further observations and clinical correlations. Neuroradiology 1988;30:379-84.
7 Roach ES. Tuberous sclerosis: function follows form. J Child Neurol 1997; 12:75-6.

8 Roach ES, Williams DP, Laster DW. Magnetic resonance imaging in tuberous sclerosis. Arch Neurol 1987:44:301-3.

9 Shepherd CW, Houser OW, Gomez MR. MR findings in tuberous sclerosis complex and correlation with seizure development and mental impairment. AJNR Am J Neuroradiol 1995; 16:149-55.

10 Jambaque I, Cusmai R, Curatolo $P$, et al. Neuropsychological aspects of tuberous sclerosis in relation to epilepsy and MRI findings. Dev Med Child Neurol 1991;33:698-705.

11 Menor F, Marti-Bonmati L, Mulas F, et al. Neuroimaging in tuberous sclerosis: a clinicoradiological evaluation in pediatric patients. Pediatr Radiol 1992;22:485-9.

12 Goodman M, Lamm SH, Engel A, et al. Cortical tuber count: a biomarker indicating neurologic severity of tuberous sclerosis complex. J Child Neurol 1997; 12:85-90.

13 Jambaque I, Chiron C, Dumas C, et al. Mental and behavioural outcome of infantile epilepsy treated by vigabatrin in tuberous sclerosis patients. Epilepsy Res 2000;38:151-60.

14 Jozwiak S, Goodman M, Lamm SH. Poor mental development in patients with tuberous sclerosis complex: clinical risk factors. Arch Neurol 1998; 55:379-84.

15 Riikonen R, Simell O. Tuberous sclerosis and infantile spasms. Dev Med Child Neurol 1990;32:203-9.

16 Illingworth RS. Sudden mental deterioration with convulsions in infancy. Arch Dis Child 1955:30:529-37.

17 Curatolo P, Cusmai R, Cortesi F, et al. Neuropsychiatric aspects of tuberous sclerosis. Ann N Y Acad Sci 1991;615:8-16.

18 Hunt A. Tuberous sclerosis: a survey of 97 cases. I: Seizures, pertussis immunisation and handicap Dev Med Child Neurol 1983;25:346-9.

19 Nakano C, Kousaku O, Takeshita K. [Mental development of tuberous sclerosis]. Rinsho Shinkeigaku 1983;23:228-34.

20 Nixon JR, Miller GM, Okazaki H, et al. Cerebral tuberous sclerosis: postmortem magnetic resonance imaging and pathologic anatomy. Mayo Clin Proc 1989;64:305-11.

21 Lane VW, Samples JM. Tuberous sclerosis: case study of early seizure control and subsequent normal development. J Autism Dev Disord $1984 ; 14: 423-7$.

\section{IMAGES IN PAEDIATRICS}

\section{A misadventure with magnets}

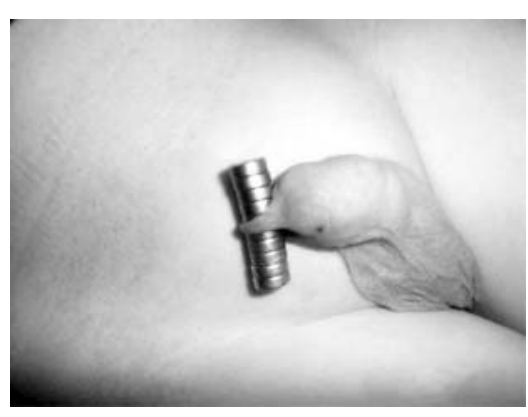

-his child tried to explore whether magnets can stick to each other in spite of skin being interposed between them. As magnets were powerful, he could not separate them for four days. It required local anaesthesia to separate them but damage to foreskin may need circumcision in future.

N B Soni, E Strydom, T Yuille Department of Paediatrics, Glan Clwyd Hospital, Rhyl LL18 5UJ, UK; nbsoni@hotmail.com 